\title{
Practices in Creating Videos with Mobile Phones
}

\author{
Arto Puikkonen \\ Nokia Research Center \\ Visiokatu 1 \\ 33720 Tampere, Finland \\ arto.puikkonen@nokia.com jonna.hakkila@nokia.com
}

\author{
Rafael Ballagas \\ Nokia Research Center \\ Palo Alto, CA, USA \\ tico.ballagas \\ @nokia.com
}

\author{
Jani Mäntyjärvi \\ VTT \\ Kaitoväylä 1 \\ 90570 Oulu, Finland \\ jani.mantyjarvi@vtt.fi
}

\begin{abstract}
Mobile phones with integrated video cameras have become ubiquitous tools that people use both to document everyday surroundings and to express themselves artistically. In this paper we report the findings of a user study on user created mobile videos, where the actions of 11 active mobile video users were documented for 2 weeks, the collected material including diaries, device logs, and altogether 255 videos. We describe the patterns related to the creation, sharing and consuming mobile videos, revealing characteristics of both context and content of the video material.
\end{abstract}

\section{Categories and Subject Descriptors}

H.1.2 [User/Machine Systems]: Human factors, H.5.1 [Multimedia Information Systems]: Video, H.5.2 [User Interfaces]: User-centered design.

\section{General Terms}

Human Factors, Verification.

\section{Keywords}

Mobile video, mobile phones, mobile multimedia, user created content, user studies.

\section{INTRODUCTION}

User created videos have become one of the prominent trends in multimedia use, and with the emergence of well-known websites for video sharing, such as YouTube [1], the content has become easier to access and distribute. With the growing popularity of cameraphones, capturing and sharing video content has become easier and faster than ever. Although technology convergence trends squeeze sophisticated video capturing technologies into a mobile phone form factor, we still have very little research on the user practices in creating mobile videos. To understand the phenomenon of capturing and sharing mobile video content, this paper aims to uncover emergent mobile video usage trends by examining 'in situ' usage data.

In this paper we focus on describing the overall process of video creation in everyday life settings. We analyze the practices in creating mobile videos on the basis of GEMS model [2], e.g. how people Get, Enjoy, Maintain and Share video content, in order to characterize the nature of mobile video composition today. This

Copyright is held by the author/owner(s). MobileHCI'09, September 15 - 18, 2009, Bonn, Germany. ACM 978-1-60558-281-8. analysis helps us identify patterns of usage that we could discuss with users during the subsequent interview process. The results of the study aim to inform the design of future video products and services for the mobile phone.

\section{RELATED WORK}

\subsection{Usage of Mobile Multimedia}

Although the history of mobile phone use is tightly coupled with the communication features, the nature of the use has broadened significantly in recent times. The mobile phone has become a multipurpose tool with an increasingly versatile feature set. Mobile multimedia use has become significant with the high adoption rate of mobile phones integrating digital cameras, high resolution color screens, enhanced audio features and software capabilities for content editing, sharing and consumption. Research on mobile multimedia has so far focused on camera phone photos [3, 4], mobile TV [5, 6, 7] and music [8, 9] rather than mobile video creation. On the other hand, the unique practices and culture around mobile phone use have provoked numerous studies. Although not focusing on mobile video creation, the phenomena appearing in the existing literature are relevant in exposing culture and practices and grounding our research to the genre of mobile multimedia usage. In the following, we take a look at the work done on the area.

The social nature of mobile phone usage has become evident not only from the basis of communication technology, such as text messaging $[10,11]$, but also with other practices related to these devices. Mobile phones serve as objects of play and artifacts for discussion [12], and in this role they can function as facilitators of social face-to-face interaction. It has also been shown that mobile phones with multimedia capabilities are used as platforms for expression and creativity. For example, Kurvinen [13] reports how multimedia messaging services (MMS) are being used e.g. for teasing friends and creative play with pictures and words. As a research concept, Salovaara [14] has designed and implemented a comic creation tool for social communication with mobile phones. Moreover, a field experiment with collaborative mobile video creation in [15] demonstrated that mobile phone video can function as a great tool for creativity, as groups of teenagers were inspired to produce and perform in their own mobile mini-movies.

Mobile phones are also used in many ways at the boundary of private and public faces of the user. It is often the content that makes the phone feel personal [16], and handling other's device may be perceived as a violation of privacy [17]. However, showing personal phone content to someone can facilitate ongoing conversation, provoke new topics, and increase the group cohesion through enjoyment of shared moments [3, 12]. Mobile phones are also being used to mark private spaces (i.e., cocooning) as a person engaged with a phone can signal that she urges to be alone rather than been disturbed by others. Here, 
mobile multimedia consumption with headphones (e.g., listening to the music or watching mobile TV) can play a role $[5,8]$.

Research on cameraphones has revealed that both functional and emotional aspects are strongly present in the practices the users have when taking photos, the latter being emphasized. In the study by Kindberg et al. [3] where a taxonomy of reasons for image capture was charted, affective aspects for individual (i.e., for personal reflection and reminiscing) and social purposes (sharing mutual experience or communicating moments to a remote friend or family member) covered $41 \%$ and $51 \%$ of the photos, respectively.

This paper is the first in-depth study focusing on everyday practices around user-created, phone-authored video content. Existing studies on mobile video have explored the differences between using heavy vs. light equipment [18] and their role alongside digital photographs and videos filmed with other devices [19]. The findings revealed that the lightweight video work, meaning the use of mobile phone for shooting the videos, included more spontaneous and 'just for fun' filming in comparison to the use of conventional video cameras. In [20], improving the mobile device UI for navigating a video timeline has been explored. Other studies related to mobile videos include collaborative live video creation [21] and the practices around video consumption (more precisely mobile TV content) [7], where for instance gift-giving practices (e.g. recording content for a friend) were exposed.

\subsection{GEMS - A tool for modeling personal media content interaction}

Users interact with content, whether it is of their own creation or from an alternate source. Interaction with media is formed of different stages that can be divided into cohesive parts. For example with video, users can record, view, listen, edit, delete, move, send and publish. In this paper, we structure our findings around the GEMS (Get, Enjoy, Maintain and Share) model for representing users' media content experience [2, 22], especially with personal media content. The model's phases of content interaction are introduced more thoroughly in the following paragraphs.

The first phase of incoming content is Get, and it can consist of, e.g., downloading, receiving, creating and purchasing. Media content does not have to be of one's own creation; it can be equally downloaded or purchased. Whatever the method of retrieval, the desired media content is delivered to the user's phone. Get is the phase that precedes any other phase and thus is always the first action in media content interaction.

Enjoy is the phase of personal utilization of content. Utilization is done for one's own pleasure. Between the phases Get and Share, interaction with media content often consists of viewing or listening to it by oneself. With video content, the viewing and listening can be done with any device that offers such functionality. Salminen et al [2] also include editing and personalizing in the Enjoy phase as it aims for the same purpose, personal enjoyment.

Maintain is the third phase of GEMS. It is the phase where content is organized, trimmed and archived. Maintaining is done with the aim of storing the content in the desired form. As enjoying had editing, maintaining includes trimming. Trimming is done for the purpose of cutting out unnecessary parts of the content and then storing and utilizing it in the desired form. Actions such as rating and synchronizing are also included in maintaining.

Share is the phase of outgoing content. Sharing consists of ways of providing the content to others. One can either share the content by merely showing it, sending it or by publishing it. Sharing is also the starting point of someone else's GEMS cycle as they Get the content from the person sharing it. Sharing does not always occur with media content; content can simply be received and personally used. Sharing is neither always the final stage of interaction as the same content can be, for example, enjoyed after sharing it

In addition to these four phases of GEMS model, Salminen et al [2] also bring forth the actions of Search and Browse. These actions can be done in any phase of the GEMS cycle; Searching for content to download, searching for content to enjoy it.

Each of the four presented phases is its own individual entity and interaction with media content can occur only in one, several or in all of the phases. Even though each phase is independent, all phases must be and always is preceded by Get, which is the initial phase for all content actions. The order of phases may also vary. Moreover, with mobile video not only mobile phone features need to be taken into account when analyzing its GEMS cycle. With connectivity methods content can be stored in alternate devices, such as computers, and enjoyed through various displays.

\section{USER STUDY}

In the study, we examined many dimensions of mobile video usage patterns. We analyzed the content of the recorded videos, the recording features used, and the practices users have in editing, sharing and storing their video clips on the mobile phone. In addition, we took a look at the activity timeline - do actions like editing and sharing typically take place immediately after recording, or are they postponed for a more convenient time? Finally, we examine the bottlenecks in the overall use.

\subsection{Study Setting}

The study was carried out in 2008 from July to September. For the study, 12 participants were recruited, of whom all were from Finland and from various occupational backgrounds. We gave each participant a Nokia N95 mobile phone for 2 weeks. The N95 included an integrated 5 megapixel camera capable of capturing video at a rate of 30 frames per second.

To gather exact information about practices and use of features, the given phones included activity-logging software. The logger was used to gather and log all key presses and access to media within the phones' camera application, gallery application and video editor application. All logs included timestamps. For the sake of privacy and practicality, activity outside these applications was not logged. This data was used to verify the interview and diary data and to understand the way the device was used.

The participants were also provided with a small paper diary to fill in during their 2 weeks of use. The diary was mainly meant to be filled after filming and included clarifying questions about the 
reasons for filming, the target group of the video, level of planning, the intentions after filming and experienced limitations of the device. By using the time stamps of the keystroke data from the activity logger, we could compare the planned time and the actual time of execution. The diary also provided a section for each time files were transferred from the phone to another location as well as a section for stating times when filming was desired, but was not possible. In addition to these the users were given no instructions on what to do besides continue living a normal life.

At the end of the 2 weeks, we collected all diaries, mobile phones (with logger data and videos). Due to privacy and confidentiality restrictions, the participant could refuse to give any specific clip(s). This was allowed to account for private situations, such as a video containing confidential material that the participant is not allowed to share. However, the participants were still asked to describe in the diary the nature of content in the excluded videos and their intended audience.

After gathering all the data, we did a preliminary analysis of the data to inform interviews with participants. The recorded individual interviews were designed to uncover the problems and limitations experienced during video recording, the user's goal in shooting the video, the target audience, as well as storing, sharing and subsequent use of the video.

We carefully considered whether to allow participants to use their own phone or to provide them with phones for the study. Ultimately, we decided to provide mobile phones to enable use of an activity logger application, which works only with certain phone models. Also, by having the same phone with all participants we could ensure that the results were more comparable between each other. As the participants were heavy users of mobile phones with earlier experience of several models, we assumed that the change of the phone model would not limit their habits with mobile video shooting. All participants were also accustomed users of Nokia's mobile phones, most also being current users. Despite most having different mobile phone models than the given one, the features in the N95 do not differ much from the phones the users have or have used. Some of the mobile phones do not provide all the features that the N95 provides, but this was not seen as a problem as prior to the study period behavior was charted to find out what the users did before and what they did during the study.

\subsection{Participants}

We targeted participants who were already actively recording mobile videos. The study participants were recruited through on the online screening survey. The online survey was circulated on Finnish online message boards, which focus on video filming or have members who belong to social groups that actively make videos. Also popular university bulleting boards were used to promote the survey.

203 individuals responded to the survey, out of which 178 were male and only 25 female. Also out of the 203, 83 used a mobile phone, 78 a digital pocket camera and 130 a video camera for filming videos. As the study was focused on active video shooters using a mobile phone integrated video camera, the participants were selected based on their frequency of using a mobile phone for filming videos. The threshold of "being active" was considered to be filming a video more than once a week on average. Out of the 203 that filled in the survey, only 15 met this requirement (12 male, 3 female). Also as the participants would be provided with a phone and would not use their own, their level of experience with a Nokia phone was screened. The selection to choose only "active" users was made as active users have faced the largest amount of obstacles. And also, their knowledge of not just about the obstacles, but also about the benefits is greater than the knowledge of the casual user.

Based on the screening survey, twelve participants (10 male, 2 female) were selected for the study, see Tables 1 and 2 for details. The average age of the participants was 30 years, ages varying from 16 to 45 . Participants were from a number of Finnish cities and represented a variety of backgrounds. The selected 12 participants had actively used a Nokia mobile phone and its integrated video camera. And as the applications for video filming in Nokia's mobile phones are in many ways consistent, by this it could be verified that using the phone and its video features would not be a problem for the participants. All the mobile phones owned by the participants have a zoom, which is digital, but none have optical zoom. Also all devices provide connectivity possibilities with Bluetooth and USB. During the study user 10 dropped out and user 9 only provided part of the requested data outside of videoclips.

Table 1. Details about the participants

\begin{tabular}{|c|c|c|l|}
\hline User & Gender & Age & Devices used for filming videos \\
\hline 1 & M & 26 & Mobile, Digital camera \\
\hline 2 & M & 26 & Mobile, Video camera, digital camera \\
\hline 3 & F & 29 & Mobile, Digital camera \\
\hline 4 & F & 16 & Mobile, Video camera, digital camera \\
\hline 5 & M & 45 & Mobile, Video camera, digital camera \\
\hline 6 & M & 27 & Mobile, Digital camera \\
\hline 7 & M & 26 & Mobile, Digital camera \\
\hline 8 & M & 33 & Mobile \\
\hline 9 & M & 36 & Mobile \\
\hline 10 & M & 28 & Mobile, Digital camera \\
\hline 11 & M & 38 & Mobile, Video camera \\
\hline 12 & M & 29 & Mobile, Digital camera \\
\hline
\end{tabular}

\section{RESULTED VIDEOS}

The total number of videos resulted from the study was 266 , out of which, three users (Participants \#1, 4, 11) removed a combination of 11 videos as they were perceived indifferent or not good enough, making the total amount of created and saved videos 255 . Ten of these were accidental shootings, created by 6 users and filmed when trying to take a photograph, making the total amount of videos created, stored and analyzed 245 . The number of videos by each participant is illustrated in Fig 1. The length of the videos was predominantly under one minute (202) or 1-2 minutes (30). 
Table 2. Participant mobile phone experience. A: Occasions of shooting videos with a mobile phone/week; B. Experience with mobile phone videos (years); C. How often changing phone models (years)

\begin{tabular}{|c|c|c|c|c|c|}
\hline User & Gender & $\begin{array}{c}\text { Current mobile } \\
\text { phone }\end{array}$ & A. & B. & C. \\
\hline 1 & $\mathrm{M}$ & Nokia E61i & 2 & 1 & 2 \\
\hline 2 & $\mathrm{M}$ & Nokia E51 & 2 & 5 & 1 \\
\hline 3 & $\mathrm{~F}$ & Nokia 6233 & 4 & 0.6 & 2 \\
\hline 4 & $\mathrm{~F}$ & Nokia N70 & 2 & 2 & 2 \\
\hline 5 & $\mathrm{M}$ & Nokia N95 & $1-2$ & 1 & 1 \\
\hline 6 & $\mathrm{M}$ & Nokia E65 & 2 & 3 & 1.5 \\
\hline 7 & $\mathrm{M}$ & Nokia N73 & 3 & 2 & 3 \\
\hline 8 & $\mathrm{M}$ & Sony Ericsson P1i & 4 & 3 & 1 \\
\hline 9 & $\mathrm{M}$ & (data not received) & - & - & - \\
\hline 10 & $M$ & (dropped out) & - & - & - \\
\hline 11 & $\mathrm{M}$ & Nokia E90 \& N85 & 2 & 6 & 2 \\
\hline 12 & $\mathrm{M}$ & Nokia N73 & $1-2$ & 5 & 2 \\
\hline
\end{tabular}

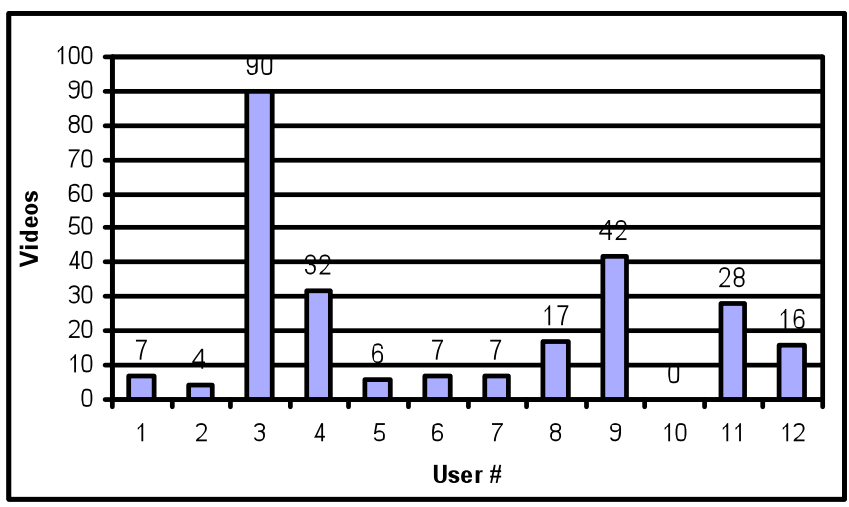

Fig. 1. Number of videos created by each participant during the study.

Interestingly, when comparing table 2 and figure 1, it seems that the amount of resulting videos drastically differs from the initially reported occasions of filming videos with a mobile. Hence, one might conclude that the self-perception of the participants were erroneous, or that behavior drastically changed for the study. However, deeper investigation shows otherwise. When analyzing on how many days per week the participants filmed during the study, it was noticed that the results had a closer resemblance to the amount initially reported, see table 3 . What the analysis on the video material also revealed was that the videos were shot in clusters, i.e. several clips immediately or shortly after another about the same target or situations. Although this typically resulted in 2-4 separate clips and a participant could make several clusters per day, the participants' perception is that they had only been 'filming once'. Only participants \#3 and \#4 stated having filmed more during the study period than on average. Here the reason for user \#3 was a trip to a foreign country during week 2 and for user \#4 it was the participation in a musical festival during week 1. For both, this can be seen in the large number of videos filmed during the study, figure 1, and the days when they filmed, table 3. This also indicates that participants' habits with video creation during the study were the same as they would have otherwise been, and speaks for the reliability of the study findings.

When preparing and during the filming itself, zoom was the only functional action taken by the participants. During the study, 8 used zoom and also 1 other stated in the interview using zoom sometimes when filming. In total, the 8 users used zoom with 71 videos out of the 234 videos they created altogether.

As to what comes to preparing for filming, it was noticed that $62 \%$ of all videos were planned. Participants stated that in some cases they know exactly what they are going to film. But for most situations the level of planning was low; users knew they would film and planned to film in a certain place, but had no idea when the actual filming would take place and what exactly would be filmed.

Table 3. Participants' video filming before the study and during the study. Prior: Initially reported occasions/clusters of shooting videos with a mobile phone/week; Week 1: Study week 1. Week 2: Study week 2.

\begin{tabular}{|c|c|c|c|}
\hline User \# & Prior & Week 1 & Week 2 \\
\hline 1 & 2 & 3 & 3 \\
\hline 2 & 2 & 3 & 1 \\
\hline 3 & 4 & 3 & 5 \\
\hline 4 & 2 & 4 & 2 \\
\hline 5 & $1-2$ & 2 & 2 \\
\hline 6 & 2 & 3 & 2 \\
\hline 7 & 3 & 1 & 1 \\
\hline 8 & 4 & 4 & 4 \\
\hline 9 & 3 & 4 & - \\
\hline 10 & - & - & 1 \\
\hline 11 & 2 & 3 & 2 \\
\hline 12 & $1-2$ & & 3 \\
\hline
\end{tabular}

We analyzed videos for their content and style. After reviewing the videos with the participants during the interview, several different categorical clusters of videos emerged (see fig. 2). Most frequently the users filmed animal and scenery videos with $5 / 11$ participants filming each type of videos. Humorous videos were 

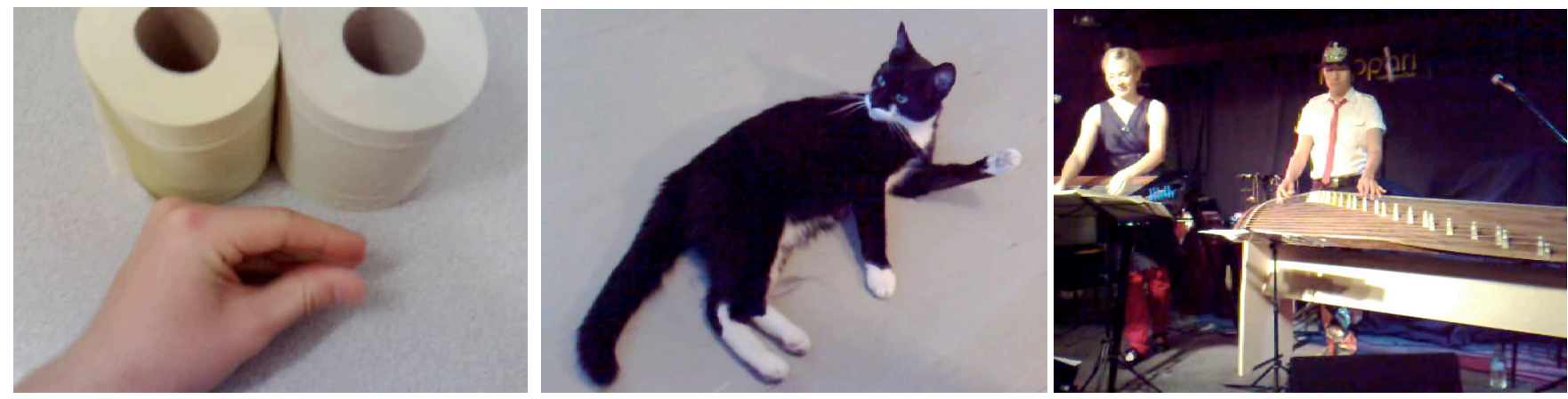

Figure 2. Screenshots from different categories of user created videos: Humor, Animal, Performance

also present with 4/11 filming such material and performances were shot by $3 / 11$ users. Other styles for video were objects of personal interest, a situation/event with social elements (both with 2 participants), work and creating art (by 1 participant).

\section{RESULTS}

In the following, we look at the findings highlighting the salient trends that emerged from the collected data in the analysis in the framework of GEMS model.

\subsection{Get - Creating Mobile Videos}

\subsubsection{Selecting the device for use}

Prior to buying a mobile phone, 5 users $(\# 1,5,7,8,11)$ stated that they used online reviews or other online forums on mobile phones to get either an overview or a complete look on the mobile phone's features (especially specifications for the camera). One user (\#7) seeks information from friends. Four (\#3, 4, 6, 12) claimed that they did not seek recommendations from anywhere before going to the shop.

Predominantly, with 6 participants stating so, the most important information about the mobile phone before buying it was the information about mega pixels in the integrated camera. For 3 (\#1, 2,3 ) the main criteria was that the mobile phone at least had a camera for filming videos. When the participants were asked about their interests in other features beyond the camera, none claimed to be interested in them before buying. The reasons for this were either not knowing how to use them, or not needing them in the mobile. Also, the features that were desired but not available were a place for a tripod (5\#) and optical zoom (\#11).

The participants buy a new mobile phone typically every 1-2 years, see table 3. During such time period, the video quality the participants experienced elsewhere increased, increasing the need for a better video quality on one's own mobile phone. The comparison between the perceived quality from one's own phone and from other newer phones has a negative impact on the frequency of use. "In the beginning you tend to use it more, but then you get discouraged when you notice just how poor the quality is." (\#11)

\subsubsection{Shooting in Action}

As can be seen table 1, the majority of participants own also other devices besides mobile phones for making videos, especially $8 / 12$ own a digital pocket camera. People chose to use in the dedicated camera over mobile phone on occasions when they desired an upgrade in video quality and recording functionality. This tended to be occasions when media creation is planned, and the target has some importance - "When we're going to a celebration, I take it with me." (\#11). This replacement is due to the achieved quality upgrade that the digital pocket camera (as stated by $\# 2,3,6,7,12$ ) or separate video camera (as stated by $\# 2,4,11$ ) offers. In addition, user \#5 used the video camera only for work purposes, when making professional films.

Based on self-report data from the participants, the digital camera was typically used for videos once or twice per month. In everyday life, the mobile phone was perceived to offer sufficient video quality and there was no need to carry an extra device. Not surprisingly, this seemed to be emphasized when mobile phone camera resolution approached the resolution of a digital pocket camera. "I have 5 mega pixels in my digital pocket camera [...] I no longer had any need for it." (\#1)

The main pro for using a mobile phone for videos is its nature as a device that is almost always with you and quickly set up for recording. For the two participants $(\# 1,4)$ who carry a digital pocket camera almost daily with them in their bag, the most common situation for using a digital pocket camera is when the device is with you and you are not in a hurry. When in a hurry, the mobile phone is used because it can be set up for recording more quickly than the device in the bag.

As stated previously, 8 users used zoom in a total of 71 videos. Commonly among participants, attitude towards zoom was negative, despite its use, due to its impact on the resulting video quality. What the participants explained of their use of zoom was that it is the necessary evil; sometimes you have to use it due to distance from subject, but also to create life into the video itself. For the remaining 3 users, the poor video quality and lack of benefit were the stated reasons for not using zoom.

Based on the observations thus far, we can conclude that a typical situation for filming consists of sudden situations, despite being aware that one might have to film at some point. One or more clips are filmed and zoom is used when objects are distant. No editing or other manipulation is done to the video at this point. The video is primarily for self-consumption, but occasionally for others. The context is of interest to oneself and most likely to others as well.

\subsection{Maintain - Transferring and Maintaining Video Files}

After videos are created, there often comes a point when the videos are transferred from the phone to another location or device. For this purpose mobile phones provide connections via 
USB, Bluetooth, direct online upload and removable memory cards for separate memory card readers.

\subsubsection{When to Transfer}

When examining video transfers from the phone, the most evident finding was that the obstacles preventing a seamless and quick transfer of files had a great effect on the practices. Clearest indication of this was that $5 / 10$ users $(\# 2,4,6,7,12)$ stated that they most often transfer the videos from the mobile phone when the provided memory is full. This is due to the inconvenience "I'm just too lazy to do it." (\#6) and slowness of the operation. "The transfer is just so slow that I don't have the patience for it." (\#4) When the provided memory is full, the participants transfer the files as they have to. "When I'm forced." (\#7). Analyzing the logger data and diaries verified the comments made by the participants. Out of the 5 all transferred the video from the phone in the end of the study as they were "forced" to do so. User \#7 had planned to transfer some of the videos a week earlier, but failed to do so. He stated in the end interview that due to the slowness and inconvenience the transferring again moved to the point when he was forced to.

This lack of transferring behavior has an impact on filming activity when the memory card is full. "It basically goes to that a) it reduces filming b) you have to think what you are going to film and c) to think when you are going to transfer them." (\#12). Similar implications were also stated by Users \#4 and \#7.

Another implication of the inconvenience and slowness of the transfer was stated by Users \#3 and \#8, who commented in the end interview that the transferring is normally done when "I'm not in a hurry" (\#8), "Normally I'm in a hurry [when transferring the files]" (\#3). Here, the analysis of the logger data and diaries showed that both participants had planned to transfer the videos during times, when one is expected to have extra time. Both had planned to do the transferring the next weekend, but both failed to do so and ended up doing them on a later time during the following week.

Differently to others, 3 users $(\# 1,5,11)$ stated that they tried to transfer videos from the mobile phone as soon as possible. "Normally I take them when their fresh so I don't forget them there." (\#1). The logger data and diary verifies this behavior. For all three, plans were made when filming to transfer the videos during the same or the next day. For most videos the set plans were met, but for user $\# 5$ one of the plans was not met. For all three, the main reason for some videos to be transferred faster than others is a social hurry caused by need to share. "When I'm in a party, I try to transfer them the next day. [...] Some videos just lie there as they are not important." (\#5)

Generally, the elements of social hurry where heavily implied in the interviews. All 10 stated that the most stressing reason for quick transferring is the need to share a video with others. This was also the case for those that stated transferring videos normally only when forced to. When the need to share comes from oneself or from someone else, the barriers seen with transferring are still seen as troublesome, but managed due to more important reasons for transferring.

\subsubsection{Means of Transferring}

Among the participants, USB, Bluetooth, memory card reader and direct online upload were utilized in transferring the video files from phone. USB was the most frequent transfer method with 9/11 participants using it. The main reason for using USB connection was that it provided reasonably fast transfer rate and ease of initiation. What hinders this ease is the need for a separate cable. "It's irritating when you have to find the damn cable." (\#2)

For three participants, Bluetooth was a method used occasionally, but none used it as their main method of transferring. Factors that made Bluetooth less used were slow transfer rate, difficulty connecting and the technological requirements. As the transfer rate was much slower than when using USB, whenever USB is easily available it was chosen over Bluetooth. "Bluetooth is just so slow and you got the cable." (\#2). The required Bluetooth pairing created even a greater need of time. Also for many, Bluetooth transfer was simply unavailable due to the need for having a Bluetooth transmitter in the computer.

Bluetooth was perceived good for moments that were not rushed, and the transfer time would in any case be quite long. In those situations just leaving the phone beside the computer to transfer was suitable and still left the phone fully operational. Also for user \#11 Bluetooth was used whenever the computer and mobile phone were synced or in special cases when videos were transferred to the wife's phone.

A memory card reader was a transfer method used by three participants, being the main method for one. "You can't really transfer anything with the cables." (\#12). The fast transfer rate was the most important benefit, but the requirement for extra equipment that doesn't come with the phone was the greatest problem for those who did not use it.

Interestingly, only one participant (\#11) utilized the possibility of online upload. For user \#11 it gave sometimes the easiest way to upload to the service used. Most of the times, the video is transferred via a computer. Using the upload, according to the interview, offers sharing possibilities anywhere, anytime, but the quality of the services with usability and speed and privacy concerns made it seldom used: "[I need to have] control over who sees it." (\#11)

\subsubsection{Storing}

All participants responded that they stored their videos on a computer, mostly in the same folder as the photographs, although 2 users had the videos and photographs in different folders.

Data DVDs is also a method for video storing and sharing, used for larger quantities after a larger amount of media has been created and stored on the computer (4 participants). The main motivation to store content on a DVD is back-up, as computer storing in the long run was seen as unreliable and too risky and the stored content too valuable.

\subsection{Enjoy and Share - Viewing and Sharing Video Files}

After a video has been filmed, one of the following stages is viewing it. In addition to viewing the video on the mobile phone itself, transferring videos for viewing from a mobile phone to destinations, such as, a computer and a TV are available.

\subsubsection{Viewing}

The most frequent option for viewing mobile phone videos filmed by oneself was on a computer screen - Larger screen, support for home audio systems and easy access after transfer make the option to use computer so popular. All 10 interviewed participants stated using a computer screen to view the videos, 9 using it as their primary option for viewing. Two users $(\# 5,12)$ stated using a TV screen for viewing their created videos, user \#12 even as 
primarily means for that. For him, the primary reason was the even larger size of the screen and the leisure provided by other furniture, such as a sofa. As the viewing often happened from a big screen, it was highlighted that the zooming had a negative impact as the effects of zooming are more clearly seen on larger displays.

When asked about viewing the videos on the device itself, only 4 (\# 4, 5, 7, 11) participants stated using this option frequently. Others also stated that they have occasionally viewed the video right after filming, but do not do so on a regular basis. The screen size was seen to limit the behavior - "Well you don't want to show anything from that screen as it's so small." (\#2). When compared to viewing a video to viewing a picture right after filming, the video viewing was seen as taking too long and only videos that had importance were checked for quality and possible errors. The reason for showing videos on mobile phone was same for all. All 4 stated that it is on opportunity that allows you to show videos to people that wouldn't see them in any other way. "When you can't get on a computer and want to show quickly." (\#7). For user \#11, who stated showing often videos from a mobile, stated that the possibility for direct showing from a mobile phone is very important. "Almost as important as the camera itself, is the possibility to show directly from the device. "\#11). According to him, the reason for such high importance was the large amount of people with which the video is shared.

\subsubsection{Sharing}

\subsubsection{Individual and Social Aspects}

Based on the diary data and interviews, the videos do have a rather wide audience, with the emphasis on those that are close to the content creator, e.g. friends or family members. The most common audience for videos was the user him/herself and family and close relatives. Out of all the videos $25 \%$ were meant for members of the family and close relatives. Other groups that are the target audience were acquaintances (19\%) and customers $(2 \%)$. However, with the vast majority of the content, the creator $\mathrm{him} /$ herself seemed to be the main target. Out of all the videos $85 \%$ were meant for the user him/herself, either solely or together with other target audience. Although $51 \%$ of all videos were created for someone else, only 2 videos of the $51 \%$ were meant solely for them.

The interest of the other person was perceived to be vital for sharing, as apparent in the interviews. When dealing with a person who had been there, it was assumed that there was interest for sharing the content. When sharing was done with those that had not been present at the time of the filming, it was important to know whether the recipient would be interested in the content, as evident e.g. from the following statement: "I simply share just to those [people] that are actually interested in [the content]." (\#5) Even though the audience for sharing was rather wide, the reasons for sharing are commonly the same across the groups. Especially with friends and acquaintances, but also with family members and relatives, the reason most often was sharing 'because those people were also there' when the filming was done. Whether the recipient was actually visible in the videos or not did not matter in most cases. The presence of the other person(s) seemed to create the need to share the video with them.

As found out in earlier research [7, 23], also we witnessed that the technology functioned as a mediator in facilitating social, localized interaction. As an example, one of the participants filmed his son and visa versa, the action becoming a shared experience and providing means for accomplishing a task together. The same pattern was repeated with a mother and child.

\subsubsection{Target Audience - The 'Been There' Phenomenon}

Similar to earlier research for camera phone photos, the content was typically perceived to be for all the friends or family members present while shooting the clip. However, contrary to the photo taking, the clip was rarely shared or shown immediately after composing it [3].

Interestingly, mobile phone videos were often intended as evidence that the user had been in some situation in person. In our study, the phone camera was thus used as a documentation tool for social purposes, but in this case, the documentation was done for showing other people that you had been present in the situation, e.g. a rock concert, not so much for sharing a mutual experience. Showing the video to a friend later on functioned as a proof of having 'been there', even to boost the (personal) experience. Using mobile multimedia content to impress others has been reported in [7], where people used recorded mobile TV clips for this purpose.

Moreover, although the subject of filming was something in the environment, the documentation was intended to portray something about the user, not necessarily the event itself. This provides a new aspect to the use of camera phones.

\subsubsection{The Means for Sharing}

The participants use a large variety of options for sharing the videos. The most favored option for sharing is the larger screen used at home, mainly computer screens. But as this limits sharing to situations when guests are visiting at home, participants are utilizing other methods to get a larger audience. For those that do not use these methods, it makes sharing more difficult. "There is a lot [of videos] that are left without showing." (\#6)

As reaching the intended audience is a problem, methods for sending the video are heavily represented among the participants. All 10 participants use some from of sending feature provided by the internet. The most frequent one was using email. 7 participants $(\# 2,5,6,7,8,11,12)$ stated using email for sending videos. The reported benefits of email are that the counterpart does not have to be online the same time, direct receiving for the receiver and ease of use. No extra actions to receive the video makes viewing easy, as participants stated, because you can do it when it suits you. "When the file is in the computer, it goes from there." (\#11) The big downside of emailing, according to the participants, is the large file sizes and small inboxes.

Due to the conflict of large video files and small inboxes several participants use instant messaging systems for sharing. 5 participants (\#1, 2, 3, 4, 6) utilized MSN Messenger, 1 participant (\#2) used IRC and 1 participant (\#8) Skype. The reported benefits of using instant messenger for sharing were fast transfer, easy access for the counterpart, and flexibility with file size. As the file size flexibility helps with the file size problem faced with emails, the need for both to be online and the need for the other to have the same program are the downsides of instant messengers. 
"Whenever I have the opportunity [...] when the others are online." (\#1)

A third solution to adjust to the problems of emails and instant messengers is using online server space. 4 participants (\#2, 5, 7, 11) stated having access to their own personal online account on a server, which space for personal content. Using a server allows larger file sizes and does not require the counterpart to be online. But what the participants stated in the interview was that when adding something there you still have to contact and pass on the content link to the receiver, either with email or using instant messaging.

Using online communities for sharing was very limited among the participants. Out of the all, $3(\# 2,4,7)$ had uploaded personal videos to YouTube, $2(\# 4,7)$ to a Finnish online community called IRC-Galleria and 1 (\#11) using a blog. Only user 11 with the blog use was the only active user. For the others the experiences with YouTube and IRC-Galleria have been one-offs and none stated in being interested in sharing more video content in them. Interestingly, the reason for not wanting to upload videos to such popular online services seemed to be the lack of appreciation for the self-authored videos. "I just haven't filmed anything that would interest a larger audience." (\#1) For user \#11 the blog offers a platform for sharing content directly to a larger audience, but offers strict control over who gets access to the blog and information about the viewers. "How can I best control who gets in there and can I install web analytics [...] and those clearly made me choose that one." (\#11)

\section{DISCUSSION}

\subsection{GEMS Get-Enjoy-Maintain-Share}

As stated by the users, the main benefit of using a mobile phone for filming videos is its pervasive nature. One has a mobile phone with him or her almost all the time, regardless of its mobile video features. The users also stated that the mobile phone provides sufficient quality for video in everyday situations, which makes it unnecessary to carry an extra device with you. This emphasizes the current importance of the Get phase.

The use of a mobile phone for video filming seems to be, based on the results, a tension between convenience and quality. As the video quality is not as good as desired, but yet the easy access and pervasiveness make it the device used for these users. What became apparent from the results is that the pervasive nature of the mobile phone is set aside when the content becomes important and when video recording is planned. As stated, many felt that the upgrade in quality given by another device is enough to make one bring along another device in these situations.

Even though users carried these specialized devices, such as a digital pocket camera, the rapid nature of many content creation situations supports the use of a mobile phone anyway. As stated in the results, $38 \%$ of videos were unplanned and occurred in timeconstrained situations. The required fast response time makes the mobile a better choice for video recording as it is normally faster to access in a rapid situation. Also as $38 \%$ are unplanned, such a situation would require carrying along another device, which was not preferred by the majority of users.

Just as the perceived video quality decreases the number of videos filmed and hinders getting the desired kind of content, it also has a negative impact on the actual consumption (Enjoy) of videos. The users stated that all of them utilized larger displays and that the limitations of the quality are visible. Although the larger displays are the method preferred by all of the participants for consumption as the screen on the mobile phone itself is seen as too small. This preference makes the observation of lesser-than-expected quality even more apparent due to the larger screen size in comparison. The only ones who stated using the mobile phone's screen for viewing videos, said that it is for checking quality after filming for important videos or for sharing to others. What was also stated by the users was that even the quality inspection was used selectively; validating the quality of a video takes much longer than validating the success of a photograph.

The most apparent implication of using larger displays, instead of the mobile phone itself, is the fact that one has to wait until the videos are transferred on to an external device or use a direct cable connection to a display, if such is provided. And as only one of the users stated using a TV-Out cable, others are bound to the moment of transferring videos.

And the wait until transferring (Maintain) truly makes the personal enjoyment severely delayed. As it was stated by the users, for some the videos are transferred when not in a hurry. But for over a half, videos are transferred to the computer normally when the memory card is full or when there is need to share a file. None of the features of personal enjoyment break the barriers that the uncomfortable transferring process includes.

Many of the users' statements explain the nature of the problems they face when transferring files; Need for other devices, slowness of maximum transfer speed and connecting different devices. And as it is apparent from the results, none of the methods seem to truly surpass the problems that the users face. But based on the features that the problems have, a technology that requires no addition devices, is relatively fast and easy to connect, would be the killer application.

But what is breaking the barriers of transferring problems, is the need and want to share. What was common among most of the participants, the videos were transferred faster to the computer when there was a need or a must to share. What was also noticed is that the need to share breaks the barriers that the small screen on the device creates. So overall the need and must to share is breaking the barriers of use in many ways. Just as Kindberg et al [3] present in their finding for photos, videos are seen as important and for a substantial factor in the process of creating content. Videos are also shared with those that are present during the time of creation. But what is contrary to the finding of Kindberg et al, is the lack of "in the moment" sharing. As the participants stated, the sharing from a mobile is utilized for the sake of access, not the sake of the social situation.

For sharing straight from the device the main benefit was ease of sharing. With other methods users are bound to the limitations of technology and social schedules. This is also the case when sharing straight from one's own computer via a display. With sharing straight from own devices, the user does not have to wait for the counterpart to come online, no need to transfer first to a computer and no need to use sometimes complicated online services. The one sharing is there, the receiver is there, the opportunity is there. It is also a way to access those that are beyond reach with other means available. Not all target groups that the users focus on are necessarily technologically savvy; Grandparents, parents etc. But what was also stated by the users is 
that this method, though preferred, leaves many videos unshared due to it requiring such special circumstances.

To be able to share more and to a larger audience, the users use online communication to avoid the barriers created by sharing from a device. In contrary to the finding of Kindberg et al. for camera phones [3], the participants did utilize sharing for videos after the moment has passed and often such a moment was the preferred way of doing. But the reasons that make the sharing from the device easy make the online sharing difficult. There is need to wait for the counterpart to come online, there is the need to use a sometimes complicated online service and there are the limitations in size. What was also brought up by the users is the privacy of their videos. A common opinion was that the videos are just "my simple videos" as User 5 put it and with private and personal videos, the need to control one's privacy is crucial.

It can also not be assumed that the preferred method of sharing for the sender is suitable for the receiver. The users reported that they do try to take the receiver's preferences and actions needed into account when sharing online and aim for is an easy process for both ends. What needs to be taken into account in this matter is that most of these people, who are the target for sharing, are family members, friends and others who are a part of the users' lives; discretion and flexibility is needed and desired.

But what is also implied by the results is the fact that sharing is enjoying and a means to give enjoyment out of the videos after their creation. As personal enjoyment had its barriers and so had maintaining the videos, sharing was still done extensive. Not just out of pressure, but out of the fact that the users want to share the videos they have created with those that are interested in the content and desire others to see the content.

The study was designed to focus on users who actively film mobile videos, who film more than once per week. However, finding these participants was a relatively demanding task. A total of 205 participants filled in our screening survey, out which a total of 180 were male and 25 female. Only 15 of all the 205 corresponded with the criterion of filming more than once a week on a mobile. Out of the 15 , only 3 were female and 12 male. Hence the small amount of female in the study was not due to selection, but the lack of women corresponding with the criterion. The end result was 12 users as 3 were not willing to participate. In addition, in the survey 22 participants stated filming once per week on a mobile. Out of the 22 , only 1 was a female and the rest male. In total 85 out of the 205 film at all with mobile phones.

Because our study focused on users who actively film mobile videos, it most likely has an impact on the results. Due to active filming these users have more videos on their mobile phones. This most likely has an impact on the desire to share, but most of all it has an impact on maintaining the videos. Users, who film lesser amounts, do not have such amount of videos to transfer and may not get frustrated with the quality problems as soon as the active users. This is based on the notion that the active users stated to film even more in the beginning after receiving the device, but for casual filmers this extensive amount of videos is reached after a longer period of time.

But what comes to the results, we feel that the 11 participants clearly give us insight on the barriers and problems they face. Even though the amount of 11 is not too extensive, the sample was still relatively similar in use background and the end-results had definite similarities throughout the sample.

\subsection{About the Method}

Methodologically, it was perceived that the combination of device activity logs, user diaries, analyzing the created material complemented with user interviews gave a thorough picture of the practices the users had in creating mobile videos. Moreover, the data received from these different sources could be used to validate the completeness and quality of the other sources (e.g. by comparing logs and diaries).

Although one could argue that the selected phone model had some effect to the findings, the authors believe that these results apply to the broader domain of mobile video capture. The differences, both in technology and available features, between today's (decent) camera phones are relatively small, and the authors believe that these findings can be generalized over the mobile phone video creation culture. None of the features and factors studied in this study is such that the participants haven't had them in their own devices. Also as all participants were experienced users of features related to mobile video and accustomed to the feature style in the given device, we strongly believe that in the selected scope this study setting did not hinder the quality of the results. What supports these notions is that the results show no indication of changed behavior in comparison to prior behavior.

\section{CONCLUSIONS}

This paper present the results of our recent study of usage patters of mobile video created by $14-45$ year old active filmers, involving 11 participants and resulting 255 mobile phone created videos. Among all participants the mobile phone provides an everyday access to create videos and allows them to store and share their chosen moments. Our findings reveal that that the created video content was mostly targeted for the user him/herself, either solely or together with some other person(s). This is similar to ones reported in [3] for mobile phone photos, although our study indicates that the phenomenon seems to be even more emphasized with video work. Based on the results it is clear that the mobile phone is seen as an important part of self-created content and the means for storing content from all moments of life, but it was evident that also barriers, mostly technical, limited the use of mobile phones as video creation tool. The key factor for using the mobile phone for video creation was that the phone was always with and thus easily available.

One of the most prominent findings of our study was that the barriers shape the usage practices with user created mobile phone videos. There are several types of limitations, which have effect on the user behavior - technical, social, and the ones set by the context of the use, the first one observed to set the biggest barriers. Probably the clearest example here was the inadequate data transfer speed in handling the videos for storing and sharing. This made the transfer of the videos uncomfortable. Even more, to feeling created a mental barrier to start doing it, which lead users to either postpone the action until the last moment, a phenomenon which dominated the usage behavior in storing the material. Although the transfer speed is a well-known limitation of the current mobile technology, the evidence of its effect to the usage practices was surprisingly strong. 
Another clear barrier relating to the shooting was the time pressure - either social or physical - in the moment of shooting the film. This was connected to the slowness of the gadget especially when starting the recording. Opening the application and operating with the device settings was perceived too slow, and fast moving objects could not be filmed as accurately or with sufficient quality. Also, deleting accidental or unsuccessful shots was perceived too cumbersome in the moment, and often left undeleted, followed by a more successful second trial. The screen size effected to the consuming of the video clips and the use of the application settings. The videos were mostly made with consumption on a big screen in mind, and because of that, the resolution was almost always set to the highest option possible. Whereas earlier research on camera phone photos emphasizes sharing and showing the photos from the device [1] where a lower resolution might be suitable, our study indicates that this is not a prominent case with mobile videos.

This paper contributes to give a better understanding on users, who actively create videos with mobile phone integrated cameras and expresses the needs and barriers they face when trying to reach their aim. The focus of this paper has been in describing the overall practices what people have in creating video content on mobile phones. As this has been so far an unexplored field, the authors believe that this study has exposed valuable findings for researchers investigating the adoption of mobile multimedia as well as for developers of related applications. For future work the authors aim to dive more deeply into the content of the user created videos.

\section{REFERENCES}

[1] Youtube. Available: www.youtube.com.

[2] Salminen, I. Lehikoinen, J. Huuskonen, P. Developing An Extensible Mobile Metadata Ontology. In Proceedings IASTED conference of Software Engineering and Applications (SEA), ACTA Press, 2005, pp. 266-272.

[3] Kindberg, T, Spasojevic, M, Fleck, R, \& Sellen, A. (2005). The Ubiquitous Camera: An In-Depth Study on Camera Phone Use. Pervasive Computing, April-June 2005, 42-50

[4] Sarvas, R., Oulasvirta, A., Jacucci, G., Building Social Discourse Aroung Mobile Photos - A System Perspective. In Proceedings of MobileHCI'05

[5] O'Hara, K., Mitchell, A. S., Vorbau A. Consuming Video on Mobile Devices. In Proc. CHI 2007, 857-866.

[6] Repo, P., Hyvönen, K., Pantzar, M., Timonen, P. Mobile Video. National Consumer Research Center, Publications 2003:5.

[7] Harper, R., Regan, T., Izadi, S., Mosawi, K. A., Rouncefield, M., and Rubens, S. 2007. Trafficking: design for the viral exchange of TV content on mobile phones. In Proceedings of the 9th international Conference on Human Computer interaction with Mobile Devices and Services (MobileHCI '07) vol. 309. ACM, New York, NY, 249-256.
[8] Nettamo, E., Nirhamo, M., Häkkilä, J. A Cross-Cultural Study of Mobile Music - Retrieval, Management and Consumption. In Proc. OZCHI 2006, 87-94

[9] Voida, A., Grinter, R. E., Ducheneaut, N., Edwards, W. K., Newman, M. W. Listening In: Practices Surrounding iTunes Music Sharing. In Proc. of CHI 2005, ACM Press (2005), 191-200

[10] Grinter., B., and Eldridge, M. (2003). Wan2tlk?: Everyday Text Messaging. In Proc. CHI 2003, ACM Press (2003).

[11] Barkhuus, L. Why Everyone Loves to Text Message: Social Management with SMS. In Proc. of GOUP'05, 324-325, 2005

[12] Taylor, A. S. and Harper, R. 2003. The Gift of the Gab?: A Design Oriented Sociology of Young People's Use of Mobiles. Comput. Supported Coop. Work 12, 3 (Jul. 2003), 267-296

[13] Kurvinen, E. Only when miss universe snatches me: teasing in MMS messaging. In Proceedings of 2003 international conference on Designing pleasurable products and interfaces (DPPI'03).

[14] Salovaara, A. Appropriation of a MMS-Based Comic Creator: From System Functionalities to Resources for Action. In Proc. CHI 2007, 1117-1126.

[15] Puikkonen, A. Ventä, L. Beekhuyzen, J. Häkkilä, J. Playing, Performing, Reporting - A Case Study of Mobile Minimovies Composed by Teenage Girls. OZCHI 2008.

[16] Ventä L., Ahtinen A., Ramiah S., Isomursu M. "My phone is a part of my soul" - How People Bond with Their Mobile Phones. In Proc. of UBICOMM 2008, Sep 2008

[17] Häkkilä, J., Chatfield, C. "It's like if you opened someone else's letter. "User perceived privacy and social practices with SMS communication. In Proceedings of MobileHCI'05. ACM Press 2005.

[18] Kirk, D., Sellen, A., Harper, R., Wood, K. Understanding Videowork. In Proc. CHI 2007, 61-70.

[19] Lehmuskallio, A. and Sarvas, R. Snapshot Video: Everyday Photographers Taking Short Video-Clips. In Proc. of NordiCHI Oct 2008, 257-265.

[20] Huerst, W. Mobile Video - The Next Killer Application or Not? In MobileHCI'08 workshop on Mobile Multimedia Content Creation and Use. Amsterdam, Sep 2008.

[21] Engström, A., Esbjörnsson, M. Juhlin, O. Mobile Collaborative Live Video Mixing. In Proceedings of MobileHCI'08, 157-166. ACM Press 2008.

[22] Lehikoinen, J. Aaltonen, A. Huuskonen, P. Salminen, I. Personal Content Experience: Managing Digital Life in the Mobile Age. Wiley-Interscience, 2007.

[23] Brignull, H., Izadi, S., Fitzpatrick, G., Rogers, Y., and Rodden, T. 2004. The introduction of a shared interactive surface into a communal space. In Proceedings of the 2004 ACM Conference on Computer Supported Cooperative Work (CSCW '04). ACM, New York, NY, 49-58. 